\title{
Compliance With Infection Prevention Guidelines By Health Care Workers At Ronald Ross General Hospital Mufulira District
}

\author{
${ }^{1 *}$ Katowa P. Mukwato, ${ }^{1}$ C.M. Ngoma, ${ }^{2}$ M. Maimbolwa \\ ${ }^{1}$ Department of Post Basic Nursing, School of Medicine, University of Zambia, Lusaka, Zambia \\ ${ }^{2}$ School of Medicine, University of Zambia, Lusaka, Zambia
}

\begin{abstract}
ABSRACT
Objective: To determine the level of health-care workers' compliance with Infection Prevention Guidelines and identify factors that influence compliance at Ronald Ross General Hospital, Mufulira District.
\end{abstract}

Methods: A quantitative study was carried out in 2007. Convenient sampling method was used. Data was obtained using a self administered interview schedule and an observation checklist. A total of 77 health care workers who included Doctors, Registered Midwives and Nurses, Enrolled Midwives and Nurses, clinical Officers, Laboratory Technicians and physiotherapists took part in the study. Additionally, 40 out of the 77 interviewed health workers were observed carrying out at least one procedure requiring compliance with the Infection Prevention (IP) guidelines.

Results: The study revealed that, high compliance was associated with inclusion of Guidelines in the Curricular, high knowledge of infection prevention/ hospital acquired infections, positive attitude towards infection prevention and availability of materials for infection prevention. The study further reviewed revealed varied levels of compliance on different components of infection prevention. The highest level of compliance ( $100 \%$ ) was with single use of needles and syringes while the lowest (35.1) was with decontamination of needles and syringes with $0.5 \%$

\section{Corresponding author:}

Katowa P. Mukwato,

Lecturer, Department of Post Basic Nursing,

School of Medicine, University of Zambia.

Email: Mukwatop@yahoo.com chlorine solution prior to disposal. Compliance with hand hygiene was moderate $(61 \%)$.

Conclusion: The study findings suggest a need for inclusion of Infection Prevention Guidelines in the health workers' curricular, provision of in-service training in infection prevention protocols and improvements in the supply of materials for infection prevention.

\section{INTRODUCTION}

Nosocomial infections are acquired during hospitalization. They are caused by Candida albicans, Escherichia coli, hepatitis viruses, herpes zoster virus, pseudomonas and staphylococcus. These pathogens are transmitted from one person to another through direct or indirect contact ${ }^{1}$. At any one time, $10 \%$ of in-patients have a Hospital Acquired Infection (HAI) 2. Nosocomial infection rates range from $1 \%$ in Europe and America to more than $40 \%$ in some parts of Asia, Latin America and Sub-Saharan Africa ${ }^{3}$. The most frequent nosocomial infections occurring in developing countries are Surgical Site Infections (SSI), urinary tract infections and lower respiratory tract infections such as pneumonia ${ }^{4}$.

These infections are usually resistant to commonly used drugs such as penicillins and they are difficult to eradicate from the health care environment. They prolong hospital stays for patients thus increasing bed occupancy and consequently consuming scarce hospital and patient resources and proving a major challenge to clinical management ${ }^{5}$. On average,

Key Words: Compliance, Infection Prevention Guidelines, health-care-worker. 
having a Surgical Site Infection (SSI) increases a patient's hospital stay by 7-10 days, with organ/space and deep incision SSI accounting for the longest stays and highest cost ${ }^{6}$.

The strain that these preventable infections puts on the health care system in terms of; prolonging patient hospitalization, treatment with expensive drugs and the use of other services such as laboratory tests, $\mathrm{X}$-rays and transfusion is immeasurable yet they can be prevented simply by adhering to simple and cheaper infection prevention and control methods ${ }^{7}$.

In Zambia, nosocomial infections are still a major challenge as demonstrated by the increase in wound infections among patients with ceaserian section which is $30 \%{ }^{8}$. It is against this background that, the Government of Zambia through the Ministry of Health and its co-operating partners developed the Zambia Infection Prevention Guidelines in 2003. This was after a realization that infection prevention was a critical component of quality health care. The infection prevention guidelines specifies the infection prevention principles which are:

- Consider every person (client or staff ) infectious

- Wash hands-the most practical procedure for preventing cross contamination

- Wear gloves before touching any thing wet broken skin, mucous membranes, blood, body fluids, secretion or excretion or before touching soiled instruments and other items.

- Use barriers- Personal Protective Equipments (PPE) such as protective goggles, face mask and aprons if splashes or spills of blood or body fluids secretions or excretions are anticipated.

- Use safe work practices, such as not recapping or bending needles, safely passing sharp instruments, and disposing sharps in a puncture proof container

- Process instruments and other items that come into contact with blood, body fluids, secretions or excretions

- Dispose contaminated instruments and contaminated waste thoroughly and properly and

- Isolate patients only if secretions or excretions cannot be contained ${ }^{8}$

Ronald Ross General Hospital, is one of the hospitals in Zambia that benefited from John Hopkins Information Education on Gynaecology and Obstetric
(JHPIEGO) in terms of training of health workers in IP guidelines. Despite the hospital staff receiving training, the hospital was still recording rates as high as $33 \%$ of post operative caesarean section wound infections (elective and emergency) $)^{9}$ against World health Organization(WHO) acceptable postoperative wound infection rate of $5 \%$.

\section{LITERATURE REVIEW}

Cleansing heavily contaminated hands with an antiseptic before patient contact can reduce nosocomial transmission of contagious diseases ${ }^{11}$. This evidence was provided for some 150 years ago ${ }^{11}$. In a review article ${ }^{11}$ Semmelweis observed that mortality rate from puerperal fever was high (16\%) before May 1847 in a clinic where doctors and student doctors provided care to women in labour despite washing hands with soap and water before entering the obstetric clinic. Semmelweis (1847) postulated that the high rates of puerperal fever was caused by "cadaverous" particles transmitted from the autopsy room to the obstetric ward via the hands of students and doctors. In May 1847, Semmelweis insisted that doctors and students scrub their hands in a chlorinated lime solution before every physical examination. Consequently the mortality rate in that clinic dropped from $16 \%$ to $3.06 \%$ in the remaining 7 months of 1847.

A report ${ }^{12}$ indicated that mortality rate secondary to burns infection was lowest in wards that were situated on the top floor probably due to minimal movements and good ventilation. This report emphasized the importance of regulating traffic flow and activity patterns a component of infection prevention. Controlling of traffic and activity patterns in a ward helps in minimizing the number of microorganisms present in the environment, as the number of microorganisms in a designated area tends to be related to the number of people present and their activity ${ }^{8,13}$.

Avalability of IP materials has been cited as important determinants of compliance ${ }^{13}$. A study conducted ${ }^{14}$ reported that inadequate supply of gloves in southern province based health facilities lead to incorrect routines, for example, the average number of vaginal examinations for each woman at the University Teaching Hospital where supply of 
gloves was adequate was 3.5 compared to 2 in Southern Province based health facilities. In another study conducted ${ }^{15}$ it was reported that general hygienic measures taken in hospitals to reduce the risk of HIV infection were insufficient and that many inadequacies stemmed from lack of supplies. Another factor that has been associated with compliance is incorporation of IP Guidelines in the health careworkers' curricular and in-service training on IP protocols $^{16}$.

\section{METHODS}

\section{Design}

A descriptive case study design was used in which in-depth description of the IP practices of health care workers at the study site was done using data obtained through interview schedules and observation checklists.

\section{Study Participants}

A convenient sample of 77 different categories of health care-workers was recruited. The inclusion criteria were working in a medical, surgical, obstetrics, operating theatre and the laboratory department, had worked for at least one month at the institution and was willing to participate in the study. This population was targeted because they are in contact with the majority of patients attended to at the institution and their IP practices can either minimize or perpetuate the transmission of nosocomial infections.

\section{Data collection}

Data was collected using a self administered interview schedule with both open and closed ended questions. Data on demographics which included age, sex, profession, number of years in service were obtained to assess their influence on compliance. Data was also obtained on the knowledge of infection prevention and hospital acquired infections, attitude towards infection prevention (using the Likert scale) and on the availability and access to materials and supplies for IP. Using an observation checklist, 40 out of the 77 interviewed health care workers were observed performing at least one procedure requiring compliance with IP guidelines.

\section{Ethical consideration}

Ethical approval was received from the Research Ethics Committee of the University of Zambia. Permission to conduct the study was sought from the Executive Director of the Hospital. All respondents consented before participating in the study.

\section{Data analysis}

Data was analyzed using SPSS for Windows Version 11.5. Descriptive statistics were used for quantitative data. Frequency distribution of variables were calculated. Chi-square was used to test association between the independent variables (Knowledge of IP and HAI, staff attitude towards infection prevention and control, availability and access to materials/supplies for IP, and management support towards the implementation of the IP program) and the outcome. (compliance with Infection Prevention guidelines). The cut-off point for statistical significance was set at 5\% (P-value $<0.05)$.

\section{RESULTS}

\section{Demographics}

Thirty nine percent of the respondents were in the age group 21-30, while the least (5.2\%) were between 41-50. Professionally, the majority (35.1\%) were Enrolled Nurses. Almost half (49.4\%) had worked for less than five (5) years. More than half (59.9) were working in medical wards with the least $2.6 \%$ working in the laboratory department.

\section{Knowledge of Infection of Prevention/Hospital Acquired Infections}

The majority (95\%) of the subjects indicated that they had heard about Hospital Acquired Infection(HAI), with $(86 \%)$ having heard of Universal Precautions (UP) or Infection Prevention (IP) guidelines. Almost two thirds $(63.6 \%)$ indicated that UP/IP were part of their training curriculum. The commonest known HAI was Pneumonia while the commonest known UP/IP guideline was hand hygiene. All (100\%) who highly complied with Guidelines had at least heard about IP/UP, although the relationship was not significant (Corrected Chisquare 2.792, df2, P-value 0.143, table 2. There was however an association between inclusion of guidelines in the curricular and compliance as $88.9 \%$ 
of respondents who highly complied with guidelines had IP/UP included in their training curricular.

The commonest known mode of transmission for HAI was contact with blood and body fluids mentioned by $(24.2 \%)$ of the respondent while the least known was via needle prick injuries $9.1 \%$. Hand hygiene was mentioned by $(21.5 \%)$ of the respondents as a means of preventing HAI while only $5.7 \%$ mentioned proper disposal of medical waste. On the overall, the majority $(44.2 \%)$ had medium knowledge of IP/HAI followed by $37.7 \%$ who had low knowledge with only 18.2 having had high knowledge. Knowledge of IP/HAI were significantly related to compliance as the majority $(66.6 \%)$ of respondents who highly complied with the guidelines, had high knowledge of IP/HAI, (Corrected Chi-square 51.768, df4 p-value 0.0000), table 2.

\section{Table 1: Knowledge of Infection Prevention (IP)/Hospital Acquired infections (HAI)}

\begin{tabular}{|c|c|c|}
\hline & FREQUENCY & PERCENTAGE \\
\hline \multicolumn{3}{|l|}{ Heard of IP/UP } \\
\hline Yes & 66 & 86 \\
\hline No & 11 & 14 \\
\hline Total & 77 & 100 \\
\hline \multicolumn{3}{|l|}{ Heard of HAI } \\
\hline Yes & 73 & 95 \\
\hline No & 4 & 5 \\
\hline Total & 77 & 100 \\
\hline \multicolumn{3}{|l|}{ IP/UP as part of training } \\
\hline Yes & 49 & 63.6 \\
\hline No & 28 & 36.4 \\
\hline Total & 77 & 100 \\
\hline \multicolumn{3}{|l|}{ Mode of transmission of HAI } \\
\hline Air borne & 23 & 23.1 \\
\hline Contact with blood and body fluids & 24 & 24.2 \\
\hline Needle sticks & 9 & 9.1 \\
\hline Contaminated instruments & 17 & 17.2 \\
\hline Contaminated hands & 13 & 13.1 \\
\hline Other & 13 & 13.1 \\
\hline \multicolumn{3}{|l|}{ Prevention of HAI } \\
\hline Hand hygiene & 22 & 21.5 \\
\hline Use of personal Protective equipment & 18 & 17.6 \\
\hline Proper disposal of medical waste & 6 & 5.7 \\
\hline $\begin{array}{l}\text { Processing of instruments } \\
\text { (Decontamination, Sterilization, } \\
\text { High-level-disinfection) }\end{array}$ & 20 & 19.6 \\
\hline Isolation & 19 & 18.6 \\
\hline Others & 18 & 17.6 \\
\hline \multicolumn{3}{|l|}{ Level of knowledge on IP/HAI } \\
\hline High & 14 & 18.2 \\
\hline Medium & 34 & 44.2 \\
\hline Low & 29 & 37.7 \\
\hline Total & 77 & 100 \\
\hline
\end{tabular}

\section{Availability and access to materials for Infection Prevention}

Only $11.7 \%$ of the participants indicated that materials for IP were always available. Almost half $(47 \%)$ stated that, the materials were not easily accessible from stores, $45.5 \%$ indicated that materials were not easily accessible at ward or departmental level. When asked about access to IP guidelines within the work-place, $84.4 \%$ stated they were displayed in the work place. A significant association was found between availability of IP materials and compliance as all (100\%) of those who highly complied with guidelines indicated the materials for IP were always or mostly available, Corrected Chisquare 18.489 , df6, p-value 0.038 , table 2.

\section{Compliance with Infection Prevention Guidelines}

On the frequency of hand hygiene, $61 \%$ mentioned all the time after patient contact or after a procedure, $32.5 \%$ most of the times, $2.6 \%$ some times and $3.9 \%$ rarely. About $36.4 \%$ of the respondents used handrub for their hand hygiene followed by $27.3 \%$ who used water with non-medicated soap. Respondents were motivated to wash ands for several reasons; $41.6 \%$ for prevention of cross infection, $20.8 \%$ workload, $18.2 \%$ presence of soap and water, $9.1 \%$ and the other $9.1 \%$ were motivated by the appearance of the patient and patient's HIV status respectively.

On the use of needles and syringe, all (100\%) the respondents indicated that they never re-use any needles or syringes; similar result was obtained during the observation of routine IP practices. On the disposal of used needles and syringe, the majority $(62.3 \%)$ put used needles and syringes immediately in either puncture proof boxes where available or improvised carton boxes. Only $9.1 \%$ reported to have had a needle stick injury in the last 12 months prior to the study.

The majority (95.8\%) reported to have been using Personal Protective Equipment (PPEs) while performing various procedures. The commonest used PPE were gloves (32.4\%) while the least used were boots $(4.6 \%)$. The majority $(39.7 \%)$ were motivated to use PPEs as a means of preventing cross infection followed by $28.8 \%$ who were motivated by the availability of the PPEs. 
Of the 77 participants, only $37.7 \%$ knew the correct procedure for decontamination of soiled instrument, $57 \%$ knew the correct procedure for cleaning soiled equipment, with only $18 \%$ mentioning the correct procedure for sterilizing or high-level-disinfection of used equipment.

Table 2: Associations between compliance and hearing about UP/IP and HAI, training in UP/ IP, knowledge of IP/HAI, availability of materials for IP and Management support.

\begin{tabular}{|c|c|c|c|c|c|}
\hline \multirow[t]{3}{*}{ Variable } & \multicolumn{3}{|c|}{ Level of Compliance } & \multirow[t]{3}{*}{ Statistic } & \multirow[t]{3}{*}{ P-value } \\
\hline & $\begin{array}{l}\mathrm{n}=18 \\
\text { High }\end{array}$ & $\begin{array}{c}\mathrm{n}=43 \\
\text { Moderate }\end{array}$ & $\begin{array}{l}\mathrm{n}=16 \\
\text { Low }\end{array}$ & & \\
\hline & $\%$ & $\%$ & $\%$ & & \\
\hline Ever heard of IP/UP & & & & 2.792 & 0.143 \\
\hline Yes & 100 & 86 & 75 & & \\
\hline No & 0 & 14 & 25 & & \\
\hline $\begin{array}{l}\text { IP/UP included in } \\
\text { Training }\end{array}$ & & & & 9.750 & 0.000 \\
\hline Yes & 88.8 & 58.1 & 61 & & \\
\hline No & 11.2 & 41.8 & 39 & & \\
\hline Knowledge of IP & & & & 51.768 & 0.000 \\
\hline High & 66.6 & 4.6 & 0 & & \\
\hline Medium & 22.2 & 69.7 & 0 & & \\
\hline Low & 11.2 & 25.5 & 100 & & \\
\hline Attitude towards IP & & & & 6.480 & 0.026 \\
\hline Very good & 50 & 20.9 & 24.7 & & \\
\hline Good & 50 & 79.1 & 75.3 & & \\
\hline Poor & 0 & 0 & 0 & & \\
\hline $\begin{array}{l}\text { Availability of } \\
\text { materials for IP }\end{array}$ & & & & 18.489 & 0.038 \\
\hline Always available & 27.7 & 2.3 & 0 & & \\
\hline Mostly available & 72.7 & 72 & 50 & & \\
\hline Rarely available & 0 & 20.9 & 43.7 & & \\
\hline Not available & 0 & 4.6 & 6.2 & & \\
\hline $\begin{array}{l}\text { Access To materials } \\
\text { for IP }\end{array}$ & & & & 2.840 & 0.169 \\
\hline Easily accessible & 55.5 & 32.5 & 62.5 & & \\
\hline Not easily accessible & 44.5 & 67.5 & 37.5 & & \\
\hline Management support & & & & 4.500 & 0.065 \\
\hline Good & 11.1 & 0 & 0 & & \\
\hline Poor & 88.9 & 100 & 100 & & \\
\hline
\end{tabular}

*statistic Corrected Chi-square $\mathrm{P}$-value $<0.05$

\section{DISCUSSION}

Most (39\%) of the study participants were within the age group 21-30, with only $5.2 \%$ in the age range 41-50. Almost half (49.4\%) had worked for less than 5 years. This scenario is common in Zambian Government hospitals, which attracts mostly new graduates before they gain experience. The experienced health personnel usually leave government hospitals for greener pasture within the region or overseas. The limited number of experienced health personnel in Government Hospitals could be due to the brain drain that has affected mostly the experienced health personnel ${ }^{17}$.

Eighty six percent $(86 \%)$ of respondents had heard about IP guidelines. Several other studies have indicated that majority of health care-workers in Zambia have heard about IP ${ }^{17,18,19}$. Almost all (95\%) indicated that they had heard about HAI. Despite such as a response, when asked to give examples of HAIs, most mentioned conditions such as malaria, scabies and mumps with only $9.8 \%$ mentioning Post operative wound infections even though they were common at the institution averaging $15.5 \%$ per year $^{20}$. This implied that, a very small percentage of health care workers at the institution knew that Post operative wound infections are nosocomial.

The knowledge of HAI in terms of transmission was good, all who heard about them were able to mention at least one correct mode. The overall level of knowledge on IP/HAI was as follows; the majority $(44.2 \%)$ had medium knowledge, followed by $37.7 \%$ who had low knowledge and $18 \%$ with high knowledge. This finding is consistent with that by Munganga, ${ }^{19}$ in which the majority $(78 \%)$ of her study participants had medium knowledge. This finding suggests that more need to be done in imparting health care-workers with information on IP as knowledge of IP was found to be associated with compliance.

The results revealed varied levels of compliance across different components of IP. The highest (100\%) level of compliance was with the single use of syringes and needle while the least $(35.1 \%)$ was with decontamination of needles and syringes with $0.5 \%$ chlorine solution prior to disposal. Compliance with hand hygiene was moderate at $61 \%$. The $61 \%$ compliance was slightly lower keeping in mind the findings of a study by the Central Board of Health and the Prevention of the Medical Transmission of HIV project ${ }^{21}$ where compliance with hand hygiene was at $66 \%$. The figure was however, higher when compared to that obtained by Didier et al $1999^{7}$ where compliance with hand hygiene averaged $48 \%$.

In addition, the results of the study revealed that compliance also varied across different Hospital Departments and with different procedures. 
Compliance was significantly high in those departments such as the Operating Theatre where supplies of materials for IP were adequate compared to the Medical Department where supplies were inadequate. Ninety percent of the participants observed in the Operating Theatre (conducting or assisting an operation) complied while none of those observed in the Medical Department (administering an Injection) complied, Corrected Chi-square $=19.861$, $\mathrm{df} 4, \mathrm{p}$-value 0.000 . Similar results were reported in previous research ${ }^{14,15,22}$ where lack of necessary equipment and protective clothing were the major reasons why health care-workers did not adhere to Universal Precaution.

The attitude of health workers towards IP was positive, as the majority $92.2 \%$ strongly agreed that IP was very important. Similarly, 90.9\% strongly agreed that IP does not only benefit the hospital, but the client, staff and community at large. This implied that the relative low level of compliance established at the institution during the observation of routine IP practices was not due to negative attitude.

\section{CONCLUSION}

The study demonstrated that levels of compliance vary across different components of Infection Prevention, Departments and type of procedure. Factors associated with high compliance were, inclusion of Infection Prevention Guidelines in training, high Knowledge of Infection Prevention Guidelines/Hospital Acquired Infections, positive attitude towards Infection Prevention and adequate supplies of Infection Prevention materials.

\section{Limitation of the study}

1. The small sample size and the convenient sampling method used in this study limits the generalization of findings to other settings.

2. Only 40 out of the 77 interviewed candidates were observed on their infection prevention practices. This could have biased the results since some of the participants who were not observed could have had some peculiar IP practices

\section{REFERENCES}

1. Anderson, N.K. and Anderson, L.E. 1995. Mosby's Pocket Dictionary of Nursing, Medicine and Professions Allied to Medicine. M Mosby, London.

2. Taylor, K., Plowman, R .and Roberts, J.A. 2001. The Challenge of Hospital Acquired Infections. National Audit Office, London.

3. Lynch, P. 1997. Infection prevention with limited Resources. ETNA communications: Chicago.

4. Emori, T. J. and Gaynes, R. P. 1993. “An overview of nosocomial infections, including the role of microbiology laboratory". Clin Microbiol Rev 6(4):428-442.

5. Plowman, R. N., Graves, M., Grifin, J. A., Roberts, A., Swan, A and Cookson, A. et al. 2000. Socio-economic Burden of hospital acquired infections, Public Health Laboratory Service. London.

6. Tietjen, L., Bossemeyer, D. and Mclntosh, N. 2003. JHPIEGO Corporation, Mary Land, United States of America.

7. Didier, P., Mourouga, P. and Pernoger, T. V. 1999. "Compliance with hand washing in a Teaching Hospital" Infection Control Programme., Ann. Intern. Med, 126-130.

8. Central Board of Health. 2003. Zambia Infection Prevention Guidelines, Lusaka, Zambia.

9. Ronald Ross General Hospital. 2006. Health Management Information System.

10. Maimbolwa, C. M. 2006, Consultancy Report on adoptation/Adaption of Infection Prevention Control (IPC) Policies and Guidelines or strengthening existing ones based on the Regional Prototype in Three countries Lesotho, Seychelles and Zambia. (Unpublished).

11. Didier, P. and Boyce, J. M. 2001. "Hand Hygiene and Patient Care: Persuing the Semmelweis Legacy". Lancent Infectious Diseases: April 9-20.

12. Chanda, D. 1995a. "Nosocomial Infections amongst Burns Patients at the University Teaching Hospital", Lusaka, Zambia. (Unpublished).

13. Russell, A. D., Hugo, W.B. and Ayliffe, G.A. 1982. Principles and practice of Disinfection, preservation and sterilization. Blackwell Scientific publication. Oxford. 
14. Maimbolwa, C. M., Arvidson, A. R.,Ng'andu, N., Sikazwe, N. and Diwan, V.K. 1997. Routine Care of Women Experiencing Normal Deliveries in Zambian Maternity Wards: APilot Study. Stockholm, Sweden.

15. Siziya, S., Tawanda, M., and. Kaona, F. 2002. "Was there evidence of occupational exposure to human immunodeficiency virus (HIV) infection among nurse-midwives 10 years into the epidemic in Zambia". African Journal of Health Sciences, 2002; 7:3-4.

16. Hamomba, L. 2006. Adherence to Universal Precautions with Reference to HIV Infection among Midwives and Trained Birth Attendants during Home and Health Centre Deliveries in Siavonga and Mazabuka Districts. Masters. Dissertation, University of Zambia. (Unpublished)

17. Churches Health Association of Zambia (CHAZ). 2007. Health worker shortage in Africa worst in the world. CHAZ Directory, Documents and Publications, Lusaka, Zambia.
18. Muchemwa, E. K. 1996. A study examining Nurses Practices towards infection Control at Kitwe Central Hospital, Lusaka, Zambia. (Unpublished)

19. Munganga, J. K. 2007. a study to determine Knowledge, Attitude and Practices of medical Doctors on Infection Control at kitwe Central Hospital, Lusaka, Zambia. (Unpublished)

20. Ronald Ross General Hospital. 2006. Infection Prevention Report.

21. Central Board of Health and the Prevention of Medical Transmission of HIV Project. 2004. Trails of Improved Practice, Lusaka, Zambia.

22. Kumar, R., Mohan, N., Seenu, V., Kumar A., Nandi, M. and Sairma, R. K. 2002. "Knowledge Attitude and practices towards HIV among Nurses in a Tertiary Care teaching Hospital; Two decades after the discovery. Journal of Communicable Diseases; 34 (4): 245-56. 